\title{
Percepção da imagem corporal e nível socioeconômico em adolescentes: revisão sistemática
}

\author{
Adolescent body image perceptions and socioeconomic status: a systematic review
}

\author{
Érico Felden Pereira ${ }^{1}$, Clarissa Stefani Teixeira² ${ }^{2}$ Bruna Dalcin Gattiboni ${ }^{3}$, Lidiane Amanda Bevilacqua ${ }^{4}$, Susana Cararo Confortin ${ }^{3}$, \\ Tatiana Rodrigues da Silva ${ }^{3}$
}

\section{RESUMO}

Objetivo: Discutir as relações entre percepção da imagem corporal e fatores socioeconômicos como renda, grau de escolaridade e etnia em adolescentes.

Fontes de dados: Artigos selecionados nas bases PubMed e SciELO, sem limite de data de publicação, com amostras de adolescentes, nos idiomas inglês, espanhol, português ou francês, utilizando os descritores: "percepção da imagem corporal", "nível socioeconômico" e "adolescentes".

Síntese dos dados: A influência do contexto socioeconômico na percepção da imagem corporal e satisfação/insatisfação com o corpo é tema relevante no entendimento da saúde dos adolescentes. Identificou-se que as relações entre imagem corporal e nível socioeconômico são complexas e os resultados dos estudos publicados não são conclusivos. As principais tendências observadas foram: jovens norteamericanos de classe baixa apresentaram maior chance de serem obesos ao contrário dos brasileiros; moças e rapazes apresentaram comportamentos diferenciados na percepção da imagem corporal, independentemente da etnia e do nível socioeconômico; moças brancas apresentaram maior insatisfação com a imagem corporal e maior busca por dietas do que moças negras, as quais parecem sofrer menos influência dos padrões de beleza em voga; jovens de menor nível socioeconômico apresentaram uma tendência a desejarem corpos maiores.
Conclusões: Considerando-se amostras estrangeiras, houve uma tendência de maiores índices de insatisfação com o corpo em adolescentes de classe socioeconômica mais privilegiada. Em amostras brasileiras, análises sobre o assunto são escassas e pesquisas são necessárias, especialmente pelo fato de a população estar passando por modificações tanto econômicas como nutricionais.

Palavras-chave: imagem corporal; fatores socioeconômicos; percepção; adolescente.

\section{ABSTRACT}

Objective: To discuss the relationships between body image perceptions and socioeconomic factors such as income, level of education, and ethnicity among adolescents.

Data source: Articles were selected from PubMed and SciELO databases, involving adolescents, without limits of publication dates, in English, Spanish, Portuguese, or French, with the following keywords: "body image perception", "socioeconomic status", and "adolescents".

Data synthesis: The influence of the socioeconomic context upon body image perceptions and satisfaction/dissatisfaction with the body is a relevant subject in understanding adolescent's health. This study identified that relationships between body image and socioeconomic levels are complex and the results of published studies are not conclusive.
Instituição: Universidade Federal do Paraná (UFPR), Curitiba, PR, Brasil ${ }^{1}$ Mestre em Educação Física pela Universidade Federal de Santa Catarina (UFSC), Florianópolis, SC, Brasil

${ }^{2}$ Mestre em Distúrbios da Comunicação Humana pela Universidade Federal de Santa Maria (UFSM), Santa Maria, RS, Brasil

${ }^{3}$ Educadora Física pela UFSM, Santa Maria, RS, Brasil

${ }^{4}$ Especialista em Atividade Física, Desempenho Motor e Saúde pela UFSM, Santa Maria RS, Brasil

\author{
Endereço para correspondência: \\ Érico Felden Pereira \\ Rua Santo Antonio, 270 \\ CEP 83070-179 - São José dos Pinhais/PR \\ E-mail: ericofelden@gmail.com \\ Fonte financiadora: CAPES e CNPq \\ Conflito de interesse: nada a declarar
}

Recebido em: 31/3/2010

Aprovado em: 13/10/2010 
The main findings are: North-American lower class youths present greater chances for obesity, but this is not true in Brazil; girls and boys have different behaviors concerning body image perceptions, despite ethnicity or socioeconomic status; Caucasian girls show more dissatisfaction with their body images and greater search for diets than African American girls, who seem to suffer less influence of the current beauty patterns; youth of lower socioeconomic status presents a tendency to desire larger bodies.

Conclusions: Considering foreign samples, there was a tendency for greater levels of dissatisfaction with the body among adolescents of higher socioeconomic classes. In Brazilian samples, studies about this subject are scarce and further research is needed, especially because Brazilian population is experiencing economical and nutritional modifications.

Key-words: body image; socioeconomic factors; perception; adolescent.

\section{Introdução}

As relações e a influência do contexto socioambiental nas características biológicas e de saúde são temas cujo entendimento é primordial para ações afetivas em Saúde Coletiva. Neste contexto, investigações epidemiológicas recentes na população brasileira $^{(1,2)}$ observaram uma espécie de transição epidemiológica, na qual um aumento na prevalência de doenças crônico-degenerativas, especialmente nas populações com baixo nível socioeconômico, vem sendo identificado.

Dentre os diversos desfechos em saúde passíveis de análise sob a óptica das desigualdades em saúde, a percepção da imagem corporal apresenta-se como tema pouco explorado e sem resultados conclusivos, especialmente em amostras brasileiras $^{(3)}$. A imagem corporal refere-se a uma experiência psicológica de percepção principalmente, mas não exclusivamente, da aparência física. Trata-se de um construto multifatorial relacionado à autopercepção, atitudes, pensamentos, crenças, sentimentos e comportamentos em relação a si próprio e às outras pessoas ${ }^{(4-6)}$.

De maneira geral, os estudos têm identificado frequências crescentes de insatisfação com a imagem corporal em diferentes faixas etárias, especialmente nos adolescentes ${ }^{(3,7-9)} \mathrm{e}, \mathrm{com}$ relação à influência dos aspectos socioeconômicos nesse desfecho, os resultados apontam para diferentes perspectivas. No estudo de Pereira et $a^{(3)}$, com adolescentes de Florianópolis (SC), identificou-se uma tendência de os escolares das classes mais altas apresentarem maior insatisfação pela magreza e, das mais baixas, pelo excesso de peso. Schreiber $e t a^{(10)}$ verificaram, em um grupo de 2.379 crianças norte-americanas de nove e dez anos de idade, que as negras eram mais altas e mais pesadas do que as brancas, mas não apresentavam frequência maior de insatisfação com o peso e forma corporal após ajuste pelo índice de massa corporal (IMC).

Wang et al ${ }^{(11)}$, analisando a imagem de adolescentes australianos de diferentes níveis socioeconômicos, destacam que o acesso à mídia e, por conseguinte, uma exposição maior a um padrão de beleza magro e definido, levaria indivíduos das classes mais altas a apresentarem insatisfação maior com a imagem corporal em relação ao excesso de peso. Entretanto, Moore ${ }^{(12)}$ e Annunziato et al ${ }^{(13)}$ destacam que pessoas com melhores condições socioeconômicas estão mais propensas a iniciarem dietas e buscarem auxílio de profissionais ou empresas para controle de peso e intervenções estéticas.

Diante do exposto, considerando-se: a importância dos estudos que investiguem a relação entre a percepção de imagem corporal e o nível socioeconômico para o planejamento de ações em Saúde Coletiva; o bem-estar social e a escassez de trabalhos que sintetizem a literatura sobre essa temática, o presente estudo teve como objetivo realizar uma revisão sistemática para discutir as relações entre percepção da imagem corporal e fatores socioeconômicos em adolescentes.

\section{Procedimentos}

A revisão sistemática de literatura foi realizada por meio das bases de dados PubMed e SciELO, sem recorte de período, considerando-se artigos em inglês, espanhol, português ou francês, a partir dos descritores: "percepção da imagem corporal" ("body image"), "nível socioeconômico" ("socioeconomic status") e "adolescentes" ("adolescents"). A busca foi ampliada com a consulta da Biblioteca Virtual em Saúde na base Lilacs e as referências dos artigos selecionados. Na primeira busca, foram listados 48 artigos. A partir dessa primeira etapa, os artigos selecionados para análise deveriam preencher aos seguintes critérios de inclusão: apresentar dados originais, não serem voltados à investigação de populações com patologias específicas ou exclusivamente com gestantes e apresentarem análises de imagem corporal com adolescentes, relacionadas a indicadores socioeconômicos como renda, raça e etnia. Em termos gerais, mas não como critério de exclusão, considerou-se adolescentes os jovens com idades de dez a 
19 anos. Foram selecionadas 15 publicações para análise, sendo que 12 artigos apresentaram associações diretas entre parâmetros socioeconômicos como renda, escolaridade, raça e etnia com a imagem corporal (Quadro 1) e três publicações continham dados complementares. As duas principais causas de exclusão dos artigos listados na busca inicial foram: não apresentarem análises de imagem corporal, uso do tema apenas em contextualizações no artigo, ou estudos de pequenos grupos de adolescentes com patologias específicas.

Quadro 1 - Principais características dos estudos analisados

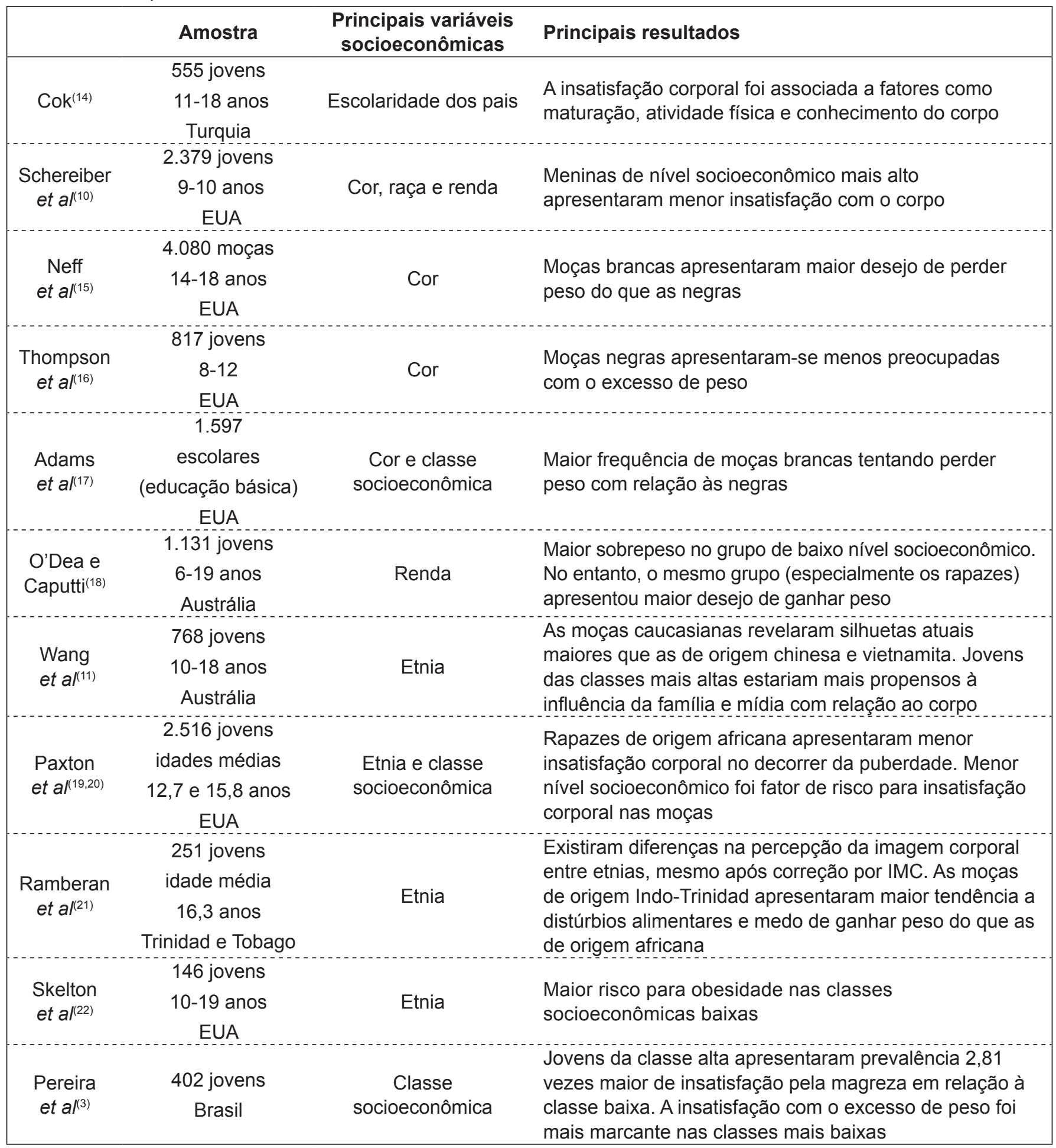


Os dados gerais dos artigos selecionados estão apresentados no Quadro 1, considerando-se o ano de publicação dos artigos como sequência.

\section{Resultados e análises}

A busca de estudos, realizada de acordo com os critérios de seleção, revelou uma lacuna de conhecimento, evidenciando um tema pouco explorado pelas literaturas nacional e internacional. Dos estudos listados, poucos abordaram diretamente as associações entre imagem corporal e nível socioeconômico e outros realizaram aproximações, considerando as desigualdades em saúde no contexto da satisfação com a imagem corporal e o estado nutricional, focando, em geral, na raça e cor dos sujeitos.

Os questionários com questões sobre satisfação com o corpo e distúrbios alimentares, as figuras de silhuetas corporais e indicadores antropométricos como o IMC foram os procedimentos mais utilizados, embora a análise de instrumentos não tenha sido foco deste texto. Evidenciaram-se, sempre que possível, resultados de análises ajustadas, já que muitos estudos não apresentaram correção por fatores de confusão.

As crianças e adolescentes têm sido foco de muitas pesquisas com imagem corporal e são apontados, juntamente com as mulheres, como um grupo mais suscetível à insatisfação corporal e aos distúrbios alimentares. Na infância, os primeiros planos corporais conscientes começam a se formar, e as relações das crianças com o ambiente apresentam grande dinâmica. Já na adolescência, um dos focos de análise recai sobre a adequação da autopercepção do corpo e a insatisfação com a mesma em relação ao real estado nutricional e forma corporal; essas relações são balizadas pelos padrões de beleza em voga e divulgados pela mídia e pela necessidade de aceitação dos jovens no seu grupo de convivência ${ }^{(9)}$.

Grande parte dos estudos com adolescentes selecionados foi conduzida com amostras norte-americanas que analisaram, em sua maioria, diferenças entre etnias e nível socioeconômico inferido pela ocupação dos pais. Nas amostras brasileiras, apenas o estudo de Pereira $e t$ al ${ }^{(3)}$ analisou com maiores detalhes a relação entre a percepção da imagem corporal e o nível socioeconômico de adolescentes.

Embora o tema tenha sido desenvolvido por um número restrito de estudos, as tendências gerais encontradas com relação aos adolescentes mostram que o comportamento de moças e rapazes, independentemente de classe social e etnia, com relação à imagem corporal são diferenciados, existindo uma tendência de maior insatisfação com o corpo no grupo feminino - especialmente quanto ao excesso de peso ${ }^{(11-13,15)}$ - e grupo masculino com relação ao desejo de terem massa muscular maior ${ }^{(3,18)}$. O'Dea e Caputi ${ }^{(18)}$ apontam que a falta de interesse na perda e maior interesse no ganho de peso entre esses rapazes deve-se ao padrão de beleza de corpos musculosos para o sexo masculino. Além disso, abordam a possibilidade de que, em muitas realidades, os fatores relacionados à cultura e etnia sejam preponderantes para a satisfação/insatisfação corporal.

As moças brancas apresentaram maior insatisfação com o seu corpo, principalmente com relação ao excesso de peso, do que moças negras, e parecem estar mais suscetíveis aos padrões de beleza impostos pela mídia ${ }^{(11,15,16,20)}$. Os fatores de confusão para essa constatação podem ser citados como a menor percepção de risco de obesidade identificada de pais e adolescentes de baixo nível socioeconômico ${ }^{(22)}$ e diferenças nos períodos de maturação de acordo com o contexto ambiental ${ }^{(23)}$.

Os jovens de nível socioeconômico baixo apresentaram uma tendência maior de apresentar sobrepeso e, por outro lado, de se definirem como magros, em estudos com amostras estrangeiras ${ }^{(19,20-22)}$. Já estudos com adolescentes brasileiros conduzidos por Pellegrini e Petroski ${ }^{(24)}$ e Pereira et al ${ }^{(3)}$ identificaram que aqueles com maior nível socioeconômico mostravam maior prevalência de sobrepeso e obesidade em relação aos adolescentes com nível econômico menos favorecido, o que pode indicar singularidades de acordo com a região de investigação e o início de uma transição epidemiológica da obesidade para classes sociais menos favorecidas, já evidenciada em países ricos.

Além disso, nos jovens com nível socioeconômico mais elevado, foi verificada uma tendência de desejo por corpos mais magros e de uso de dietas ${ }^{(11,17)}$. Em estudos como o de $\operatorname{Cok}^{(14)}$, no qual o nível socioeconômico não foi fator associado a insatisfação com a imagem corporal, houve uma tendência de fatores como sexo (maior insatisfação para moças) e maturação (maior insatisfação para jovens mais maduros e mais velhos) se apresentarem como variáveis determinantes para a satisfação/insatisfação com a imagem corporal. Além disso, conforme discutiu Kumanyika $^{(25)}$, a influência do nível socioeconômico no controle de peso e na satisfação/insatisfação com a imagem corporal é multifatorial e depende de fatores como o conhecimento e a possibilidade de comprar alimentos saudáveis, a segurança e as oportunidades para a prática de atividade física e esportes, acesso a serviços de saúde de qualidade e profissionais especializados. 
Outro ponto importante identificado nos estudos é que, embora exista uma tendência de jovens com sobrepeso, obesidade e valores maiores de IMC apresentarem frequências maiores de insatisfação com a imagem corporal ${ }^{(10,21,26)}$, existe também uma tendência crescente de adolescentes, mesmo com peso normal, estarem insatisfeitos com sua forma física, como verificado em estudos com amostras brasileiras ${ }^{(3,9)}$, devido, especialmente, à grande divulgação pela mídia de certos padrões de beleza.

A seguir, são apresentados maiores detalhes sobre os estudos com os adolescentes analisados que, além das tendências citadas, apontam, em sua maioria, para a importância de intervenções em educação e saúde já no início da adolescência, com o intuito de diminuir problemas alimentares e insatisfação com o corpo que levam a problemas psicossociais graves durante a adolescência e reflexos negativos na vida adulta.

A amostra do estudo de $\operatorname{Cok}^{(14)}$ foi formada de 269 moças e 286 rapazes de 11 a 18 anos na Turquia. O autor verificou que os rapazes apresentaram nível de satisfação com o corpo maior do que as moças $(p<0,01)$. As moças mais velhas apresentaram satisfação maior com os seios, e os rapazes mais velhos mostraram-se mais satisfeitos com seus órgãos sexuais, mas, apesar disso, ao contrário dos rapazes, nas moças a maturação mais precoce foi associada a índices maiores de insatisfação corporal. Tanto moças quanto rapazes que praticavam atividade física tinham maior satisfação com a imagem corporal do que os não praticantes $(p<0,001)$, e os rapazes que buscavam informações sobre o desenvolvimento do corpo e aparência mostraram-se mais satisfeitos com a imagem corporal $(p<0,05)$. O nível socioeconômico dos adolescentes, determinado pela escolaridade dos pais, não influenciou em sua imagem corporal.

Neff $e t a l^{(15)}$ analisaram um grupo de 4.080 adolescentes norte-americanas (1.824 negras e 2.256 brancas) de 14 a 18 anos. Os autores verificaram que mais adolescentes brancas $(41 \%)$ perceberam-se com sobrepeso do que as negras (29\%). Mais moças negras apresentaram desejo de ganhar peso, enquanto as brancas expressaram maior desejo de perder peso. Os autores discutiram a tendência de os estudos mostrarem que o nível socioeconômico é inversamente relacionado ao sobrepeso para as mulheres, já que, para os homens, o peso do corpo aumenta conforme a renda. Ainda segundo os autores, a motivação para a magreza entre as mulheres brancas é um reflexo das normas pelas quais as mulheres são julgadas na sociedade ocidental. Embora as mulheres negras estejam expostas às mesmas normas da cultura, elas parecem ter um conjunto diferente de influências e critérios de imagem do corpo. As adolescentes negras são, ainda, mais influenciadas pelos pais e adultos do que as adolescentes brancas, que são mais influenciadas por seus amigos e meios de comunicação social.

Thompson et al ${ }^{(16)}$ investigaram 817 crianças norteamericanas, brancas e negras, de oito a 12 anos. Os autores verificaram que os jovens negros apresentaram tamanhos corporais significativamente maiores do que os brancos $(p=0,01)$, mas, apesar disso, grande parte das jovens, independentemente da cor, desejava corpos mais magros. Jovens negros do sexo masculino diferem dos brancos, selecionando um maior tamanho corporal como o desejado e fazem dieta com menos frequência; as moças negras apresentaram-se menos preocupadas com o excesso de peso e se sentem menos pressionadas pela sociedade para serem magras. A análise das atitudes das crianças para a magreza constatou que elas adquirem os valores culturais de beleza antes da adolescência e que o anseio de ser magro é desejável antes da beleza. De forma geral, jovens de baixo nível socioeconômico selecionaram imagens de tamanhos maiores do que os indivíduos de alto nível socioeconômico. Os autores apontam que o início do desenvolvimento sociocultural das crianças, o sexo, a raça e o nível socioeconômico são fatores relevantes na seleção do tamanho corporal ideal e determinantes para a satisfação com o corpo. $\mathrm{Na}$ fase do desenvolvimento sociocultural em que as crianças se encontram, há fatores de sexo, raça e nível socioeconômico que são de grande influência para escolher o tamanho corporal ideal e para determinar a satisfação do tamanho corporal que desejam.

O’Dea e Caputi ${ }^{(18)}$ investigaram 1.131 crianças e adolescentes australianos de seis a 19 anos, em diferentes níveis socioeconômicos. Os autores apontaram haver interação significativa entre faixa etária, sexo e imagem corporal, sendo que moças mais velhas foram mais suscetíveis do que outros alunos a se autodefinir como muito gordas (32\%). Já os rapazes mais velhos consideram-se, em grande parte, muito magros. As crianças e adolescentes de baixo nível socioeconômico foram significativamente mais propensos a apresentar sobrepeso (26,9\% de baixo nível socioeconômico; $13,9 \%$ de médio/alto nível socioeconômico). De forma geral, os jovens de nível socioeconômico baixo, especialmente os rapazes, estavam mais propensos a se verem como muito magros, tentando ganhar peso. Os autores sugerem que os jovens de baixo nível socioeconômico, especialmente os rapazes, podem ser particularmente resistentes a influências socioculturais. 
Adams et al ${ }^{(17)}$ investigaram 1.597 jovens norte-americanas, escolares da educação básica, e identificaram que os jovens negros selecionaram um tamanho de corpo ideal significativamente maior do que os brancos, e mais jovens brancos relataram que estavam tentando perder peso em comparação aos negros. Um número maior de moças de nível socioeconômico alto estava tentando perder peso em relação às de baixo nível socioeconômico. Não foram encontradas diferenças na preocupação com a imagem corporal entre jovens brancos e negros de diferentes níveis socioeconômicos. Os autores discutiram que o desenvolvimento cultural da criança, o grau de escolaridade, o sexo, a raça e o nível socioeconômico são influentes na percepção do tamanho corporal ideal dos mesmos. Há necessidade de começar na infância a educação da saúde e bem-estar da criança, levando-se em conta a raça, sexo, idade e disparidades de nível socioeconômico.

O estudo de Schreiber et al ${ }^{(10)}$, conduzido com 2.379 norte-americanas de nove e dez anos, comparou indicadores de estado nutricional e imagem corporal por meio de uma escala de satisfação com o corpo e o IMC. Os autores verificaram que aproximadamente $40 \%$ das moças estavam tentando perder peso. As moças negras mostraram sinais de puberdade mais cedo e estavam menos satisfeitas com seu peso e forma do corpo quando comparadas às brancas em análises não ajustadas. Embora diferenças nas frequências de moças que gostariam de perder peso tenham sido verificadas entre brancas e negras, essas não foram significativas quando do ajuste pelo IMC. Os principais fatores associados ao desejo de perder peso foram os índices elevados de IMC, a insatisfação com a forma corporal e o fato de a mãe afirmar que a menina estava gorda. O IMC elevado esteve associado também a um maior número de tentativas de dieta. Após controle de fatores de confusão, as moças de nível socioeconômico mais alto apresentaram menor chance de estarem insatisfeitas com a imagem corporal e vontade de perder peso. Os autores destacam a importância de intervenções pediátricas e de educação em saúde com mães e adolescentes, já no início da puberdade, pelo fato de um grande percentual de moças estarem insatisfeitas com sua imagem corporal e já fazerem dietas.

No estudo de Wang et al ${ }^{(1)}$ com 768 adolescentes australianos de dez a 18 anos e de diferentes origens étnicas, verificou-se que as moças e os adolescentes mais velhos apresentaram maior desejo de emagrecer e preocupação com problemas alimentares. Os rapazes da classe baixa desejavam silhuetas maiores e as moças da classe alta desejavam silhuetas menores que as demais classes. As moças caucasianas revelaram silhuetas atuais maiores que moças de origem chinesa e vietnamita. A insatisfação entre moças e rapazes apresentou grande variação ( $43 \%$ para as moças e $12 \%$ para os rapazes). Os adolescentes cujos pais tinham trabalho de gerência/profissionais liberais apresentaram desejo de corpos mais finos do que filhos de executivos, assim como adolescentes caucasianos em comparação aos de origem chinesa e vietnamita. Os autores destacam haver relação direta entre insatisfação com a imagem corporal e distúrbios alimentares e, possivelmente, jovens de classe mais alta estão mais propensos a influências da família e da mídia com relação a um padrão de corpo ideal.

Paxton et $a^{(19,20)}$ apresentaram resultados de estudo com 2.516 jovens norte-americanos divididos em dois grupos com idades médias de 12,7 e 15,8 anos, com diferentes origens étnicas, em coorte de acompanhamento de cinco anos. Após correção por fatores de confusão como nível socioeconômico, estado nutricional, origem étnica, autoestima e depressão e dietas de pais e amigos, os principais fatores de risco para insatisfação com a imagem corporal nas moças foram índices elevados de IMC e menor nível socioeconômico, analisado pelo grau de escolaridade do chefe da família, pela participação da família em bolsas assistenciais e pelo tipo de emprego dos pais. Já no modelo ajustado para os rapazes, índices elevados de IMC, etnia mista e depressão foram fatores de risco que se mantiveram no modelo. A origem africana apresentou-se como fator de proteção para os rapazes para o aumento de insatisfação corporal com o passar da puberdade.

Paxton et al ${ }^{(19,20)}$ destacaram os maus tratos que pessoas obesas sofrem, incluindo discriminação no trabalho, exclusão social, pior atendimento médico, rejeição de admissão e financiamento dos estudos, o que justifica a grande importância do IMC como fator de risco. Os autores ainda sugeriram que mais investigações acerca da importância do nível socioeconômico na determinação da insatisfação corporal, especialmente nos rapazes, sejam realizadas e chamam a atenção para a necessidade de intervenções de educação em saúde no sentido de fortalecer a autoestima dos adolescentes e propiciar maior compreensão e aceitação de seu corpo.

Ramberan et a ${ }^{(21)}$ analisaram dados de 251 adolescentes com idade média de 16,3 anos de diferentes origens étnicas de Trinidad e Tobago. Embora os autores não tenham detalhado os aspectos culturais dos adolescentes, o estudo mostrou haver diferenças na percepção da imagem corporal nas várias etnias analisadas, mesmo após correção pelo IMC. As moças de origem Indo-Trinidad tinham maior 
tendência de apresentarem distúrbios alimentares e medo de ganhar peso do que aquelas de origem africana, mesmo que os jovens de origem africana tenham referido o desejo de possuírem corpos maiores, confirmando a tendência identificada em estudos com amostras norte-americanas de diferentes origens étnicas.

No estudo de Pereira et $a^{(3)}$ com adolescentes de nove a 15 anos do município de Florianópolis, SC, observou-se que a classe alta apresentou prevalência 2,81 vezes maior em relação à classe baixa de insatisfação pela magreza, tendência não observada em estudos com amostras norte-americanas. A insatisfação com o excesso de peso foi mais marcante nas classes mais baixas, indo de encontro aos resultados de pesquisas com amostras estrangeiras apresentados anteriormente. Assim, no caso dos estudos analisados com diferentes instrumentos, a classificação de "insatisfeito com o corpo"

\section{Referências bibliográficas}

1. Instituto Brasileiro de Geografia e Estatística. Pesquisa de Orçamentos Familiares 2002-2003. Antropometria e análise do estado nutricional de crianças e adolescentes no Brasil. Rio de Janeiro: IBGE; 2006.

2. Batista-Filho M, Miglioli TC, Santos MC. Anthropometric normality in adults: the geographical and socio-economic paradox of the nutritional transition in Brazil. Rev Bras Saude Mater Infant 2007;7:487-93.

3. Pereira EF, Graup S, Lopes AS, Borgatto AF, Daronco LS. Percepção da imagem corporal de crianças e adolescentes com diferentes níveis socioeconômicos na cidade de Florianópolis, Santa Catarina, Brasil. Rev Bras Saude Matern Infant 2009;9:253-62.

4. Cash TF. Our first Body Image birthday: a year in review. Body Image 2005;2:1-3.

5. Cash TF, Pruzinski T. Body images: development, deviance, and change. New York: Guilford Press; 1990.

6. Pereira EF, Teixeira CS, Borgatto AF, Daronco LS. Association between different anthropometric indicators and body image perceptions in active elderly. Rev Psiquiatr Clin 2009;36:54-9.

7. Vilela JE, Lamounier JA, Dellaretti Filho MA, Barros Neto JR, Horta GM. Eating disorders in school children. J Pediatr (Rio J) 2004;80:49-54.

8. ter Bogt TF, van Dorsselaer SA, Monshouwer K, Verdurmen JE, Engels RC, Vollebergh WA. Body mass index and body weight perception as risk factors for internalizing and externalizing problem behavior among adolescents. J Adolesc Health 2006;39:27-34.

9. Graup S, Pereira EF, Lopes AS, Araújo VC, Legnani RF, Borgatto AF. Associação entre a percepção da imagem corporal e indicadores antropométricos de escolares. Rev Bras Educ Fis Esp 2008;22:129-38.

10. Schreiber GB, Robins M, Striegel-Moore R, Obarzanek E, Morrison JA, Wright DJ. Weight modification efforts reported by black and white preadolescent girls: National Heart, Lung, and Blood Institute Growth and Health Study. Pediatrics 1996;98:63-70.

11. Wang Z, Byrne NM, Kenardy JA, Hills AP. Influences of ethnicity and socioeconomic status on the body dissatisfaction and eating behaviour of Australian children and adolescents. Eat Behav 2005;6:23-33.

12. Moore DC. Body image and eating behavior in adolescents. J Am Coll Nutr 1993;12:505-10.

13. Annunziato RA, Lee JN, Lowe MR. A comparison of weight-control behaviors e a subdivisão em "insatisfação pelo excesso de peso e pela magreza" podem mostrar resultados diferenciados, remetendo a limitações dos instrumentos que classificam apenas satisfação e insatisfação geral com a imagem corporal.

De forma geral, os estudos apontam para relações não conclusivas entre percepção da imagem corporal e nível socioeconômico, bem como para importantes fatores de confusão como idade, maturação, sexo, insatisfação com a magreza ou com o excesso de peso, raça, etnia e renda. Apesar disso, os autores indicam a necessidade de intervenções em educação e saúde, indo ao encontro do discutido por O'Dea e Caputi ${ }^{(18)}$ : profissionais que atuam em educação em saúde podem influenciar de forma importante o desenvolvimento saudável na infância e adolescência, incentivando melhores hábitos e uma compreensão mais ampla da imagem corporal. in African American and Caucasian women. Ethn Dis 2007;17:262-7.

14. Cok F. Body image satisfaction in Turkish adolescents. Adolescence 1990;25:409-13.

15. Neff LJ, Sargent RG, McKeown RE, Jackson KL, Valois RF. Black-white differences in body size perceptions and weight management practices among adolescent females. J Adolesc Health 1997;20:459-65.

16. Thompson SH, Corwin SJ, Sargent RG. Ideal body size beliefs and weight concerns of fourth-grade children. Int J Eat Disord 1997;21:279-84.

17. Adams K, Sargent RG, Thompson SH, Richter D, Corwin SJ, Rogan TJ. A study of body weight concerns and weight control practices of 4 th and 7 th grade adolescents. Ethn Health 2000;5:79-94.

18. O'Dea JA, Caputi P. Association between socioeconomic status, weight, age and gender, and the body image and weight control practices of 6- to 19-yearold children and adolescents. Health Educ Res 2001;16:521-32.

19. Paxton SJ, Eisenberg ME, Neumark-Sztainer D. Prospective predictors of body dissatisfaction in adolescent girls and boys: a five-year longitudinal study. Dev Psychol 2006;42:888-99.

20. Paxton SJ, Neumark-Sztainer D, Hannan PJ, Eisenberg ME. Body dissatisfaction prospectively predicts depressive mood and low self-esteem in adolescent girls and boys. J Clin Child Adolesc Psychol 2006;35:539-49.

21. Ramberan K, Austin M, Nichols S. Ethnicity, body image perception and weight-related behaviour among adolescent Females attending secondary school in Trinidad. West Indian Med J 2006;55:388-93.

22. Skelton JA, Busey SL, Havens PL. Weight and health status of inner city African American children: Perceptions of children and their parents. Body Image 2006;3:289-93.

23. Duarte MF. Physical maturation: a review with special reference to Brazilian children. Cad Saude Publica 1993;9 (Suppl 1):S71-84.

24. Pelegrini A, Petroski EL. Excesso de peso em adolescentes: prevalência e fatores associados. Rev Bras Ativ Fis Saude 2007;12:45-51.

25. Kumanyika SK. Obesity treatment in minorities. In: Wadden TA, Stunkard AJ, editors. Handbook of obesity treatment. New York: Guilford Press; 2002. p. 416-46.

26. Corseuil MW, Pelegrini A, Beck C, Petroski EL. The prevalence of body image dissatisfaction and its association with unhealthy nutritional status in adolescents. R da Educação Fisica/UEM 2009;20:25-31. 